\title{
Computing Versal Deformations
}

\author{
Jan Stevens
}

De computer is niet de steen,

maar de slijpsteen der wijzen.

Hugo Battus, Rekenen op taal (1983)

\section{CONTENTS}

Introduction

1. How to Solve the Deformation Equation

2. Deformations of Singularities

3. Smoothing Components of Curves

4. The Versal Deformation of $L_{14}^{6}$

5. Remarks

References

Acknowledgement

Electronic Availability
In recent years I have computed versal deformations of various singularities, partly by hand, but mostly with the program Macaulay. I explain here how to do these computations. As an application I discuss the smoothability of a certain curve singularity, a case I had not been to settle with general methods. As a result I find an example of a reduced curve singularity with several smoothing components.

\section{INTRODUCTION}

The study of well-chosen examples has always been an important tool in mathematics. In modern days, with powerful computers widely available, long and dull computations have become easy. This is especially useful for deformation theory, a subject in which there are hardly any general results beyond the existence of versal deformations [Schlessinger 1968; Grauert 1972]. Indeed, it seems that every imaginable pathology occurs: the versal base space is in general reducible, with components of varying dimension, including embedded components.

In recent years I have computed versal deformations of various singularities (I always use the term singularity to refer to a germ of a complex analytic space, but one may as well think of singular points of affine algebraic varieties), partly by hand, but mostly with the program Macaulay [Bayer and Stillman]. In this paper I explain how to do these computations.

As an application I discuss the solution of a problem from [Stevens 1989]. As a result I find an example (to my knowledge the first) of a reduced 
curve singularity with several smoothing components.

Spaces can be described in a variety of ways. My deformation computations use equations, and their result is a set of equations for the total space and for the base space. A description in terms of equations is only to be considered adequate if it is short; here all sorts of symbolic notations should be allowed. The maximum length is a changeable parameter, whose default value depends on the importance of the equations, the person doing the computation, the journal publishing the result.... For this article I have set the value at half a page. Although a system of equations gives a 'complete' description, natural questions like the number of irreducible components may be difficult to answer.

The concepts of deformations and versality can be formulated in very abstract terms. I recall the definitions in a version adapted to the needs of actual computation. Computing a versal deformation comes down to solving a deformation equation. Since this may be useful in other deformation contexts, I first discuss solution methods in a general, abstract set-up (the example of modules is treated in [Laudal 1982]). Such considerations can already be found in [Nijenhuis 1969; Griffiths 1965], but I develop them here to stress that the understanding of deformation theory benefits from one's thinking about the deformation equation. As an illustration of deformations of singularities I treat a toy example of a curve with an embedded component, namely the general hyperplane section of two planes meeting in one point (the simplest nonnormal surface singularity).

Computer computations are indispensible in the study of the general curve singularity of type $L_{14}^{6}$, consisting of 14 lines through the origin in $\mathbb{C}^{6}$. I wanted to know whether this curve is smoothable [Stevens 1989]. The computation shows that there are 16 smoothing components. However, it is not possible to write down a specific one-parameter deformation. Therefore I also compute the versal deformation (in negative degrees) of a special $L_{14}^{6}$ that is still 'general enough'. Its equations contain relatively few monomials. In practice this is a necessary condition to obtain useful explicit results.

\section{HOW TO SOLVE THE DEFORMATION EQUATION}

The first modern deformation theory is the Kodaira-Spencer theory of deformations of compact complex manifolds. Deformations are described by solutions of the following equation, which expresses the integrability condition:

$$
\bar{\partial} \vartheta+\frac{1}{2}[\vartheta, \vartheta]=0
$$

as equation in the Dolbeault complex computing $H^{*}(M, \Theta)$ [Kuranishi 1971, Chapter VII]. This is an instance of a general phenomenon in deformation theory: the problem is governed by a complex with a product, which makes the cohomology of the complex into a graded Lie algebra [Nijenhuis 1969; Griffiths 1965]. Laudal writes: 'It is now folklore that the hull of a deformation functor of an algebraic geometric object, in some way is determined by the appropriate cohomology of the object and its "Massey products", [Laudal 1982]. For recent work in this direction see [Goldman and Millson 1990].

I discuss methods to solve (1.1) in the abstract set-up of a complex $K^{\cdot}$ with a bracket operation, which descends to the cohomology. Think of the complex $K^{\bullet}$ as describing a deformation problem, with first-order infinitesimal deformations given by $H^{1}\left(K^{\bullet}\right)$ and obstructions lying in $H^{2}\left(K^{*}\right)$, while $H^{0}\left(K^{\bullet}\right)$ gives infinitesimal automorphisms. For $\varphi \in K^{1}$ consider the equation

$$
d \varphi+\frac{1}{2}[\varphi, \varphi]=0 .
$$

The problem is to find the 'general' solution of this equation. To give an exact meaning to this term, one can introduce a solution functor [Goldman and Millson 1990, $\S 1$ ], which leads back to the language of deformation theory à la Grothendieck.

Equation (1.2) is a very simple, quadratic, equation. To get a finite-dimensional solution (of dimension $h^{1}\left(K^{*}\right)$ ), one needs a transverse slice to 
the orbit of $K^{0}$; this involves in one way or another the implicit function theorem. Therefore one has to specify some analytic structure in which this theorem holds; for deformations of compact complex manifolds one can take the $K^{i}$ to be complex Banach spaces [Kuranishi 1971, Chapter X]. This method leads to the existence of a 'general' solution, but gives no way to compute it.

A different approach is to look for formal solutions, with a power series Ansatz. Write

$$
\varphi=t \varphi_{1}+t^{2} \varphi_{2}+t^{3} \varphi_{3}+\cdots
$$

and substitute in (1.2). In this formula $t$ is a parameter, which I use in a naive sense. Collecting powers of $t$ one finds the equations

$$
\begin{aligned}
0 & =d \varphi_{1} \\
0 & =d \varphi_{2}+\frac{1}{2}\left[\varphi_{1}, \varphi_{1}\right] \\
0 & =d \varphi_{3}+\left[\varphi_{1}, \varphi_{2}\right] \\
& \vdots \\
0 & =d \varphi_{n}+\frac{1}{2} \sum_{i=1}^{n-1}\left[\varphi_{i}, \varphi_{n-i}\right] .
\end{aligned}
$$

The first equation states that $\varphi_{1}$ is a cocycle, in accordance with the fact that the equivalence classes of first-order infinitesimal deformations are given by $H^{1}\left(K^{\bullet}\right)$. The second equation gives the primary obstruction: the condition for extending $\varphi_{1}$ is that the cocycle $\left[\varphi_{1}, \varphi_{1}\right]$ is a coboundary; in other words, if the cohomology class of $\left[\varphi_{1}, \varphi_{1}\right]$ in $H^{2}\left(K^{*}\right)$ is zero, one can find a $\varphi_{2}$, which is determined up to cocycles. The secondary obstruction is only defined if $\varphi_{2}$ can be found; one can still change the specific choice of $\varphi_{2}$, giving an indeterminacy characteristic of Massey triple products.

The procedure above tries to find a curve in the solution space, and the higher-order obstructions depend on the choices made in earlier steps. To avoid this problem, I will include the choices: I make a multivariable power series Ansatz for the 'general' solution. Let $\operatorname{dim} H^{1}\left(K^{*}\right)=\tau$, and choose representatives $\varphi_{1}, \ldots, \varphi_{\tau} \in Z^{1}\left(K^{*}\right)$ of a basis of $H^{1}\left(K^{\bullet}\right)$. Let $t=\left(t_{1}, \ldots, t_{\tau}\right)$ be the corresponding coordinates. I construct the local ring $S$ of the solution space as a quotient of $\mathbb{C}\left[[t]\right.$. Let $\mathfrak{m}_{\tau}$ be the maximal ideal of $\mathbb{C} \llbracket t \rrbracket$. Over $S_{1}:=\mathbb{C} \llbracket t \rrbracket / \mathfrak{m}_{\tau}^{2}$ one has the solution $\sum t_{i} \varphi_{i}$. To find the higher-order terms, write in multi-index notation an equation similar to (1.3):

$$
\varphi=\sum_{|\alpha|>1} t^{\alpha} \varphi_{\alpha}
$$

The primary obstruction comes from

$$
\sum_{|\alpha|=2} t^{\alpha} d \varphi_{\alpha}+\frac{1}{2} \sum_{|i|=|j|=1} t^{i} t^{j}\left[\varphi_{i}, \varphi_{j}\right]=0 .
$$

One can express the class of $\left[\varphi_{i}, \varphi_{j}\right]$ in $H^{2}\left(K^{\bullet}\right)$ in terms of a basis $\Omega_{1}, \ldots, \Omega_{s}$ of $H^{2}\left(K^{*}\right)$ as

$$
\operatorname{cl}\left(\left[\varphi_{i}, \varphi_{j}\right]\right)=\sum_{k} c_{i j}^{k} \Omega_{k}
$$

Equation (1.5) is solvable if and only if

$$
g_{2}^{(k)}:=\frac{1}{2} \sum_{|i|=|j|=1} c_{i j}^{k} t^{i} t^{j}=0
$$

for all $k$. It is possible that some (or all) $g_{2}^{(k)}$ are zero, even if $\operatorname{dim} H^{2}\left(K^{*}\right)>0$. Set

$$
\left.S_{2}:=\mathbb{C}[t]\right] /\left(g_{2}\right)+\mathfrak{m}_{\tau}^{3},
$$

where $\left(g_{2}\right)$ is an abbreviation for the vector

$$
\left(g_{2}^{(1)}, \ldots, g_{2}^{(s)}\right),
$$

and choose a basis $B_{2}$ of monomials for $\mathfrak{m}_{\tau}^{2} /\left(g_{2}\right)+$ $\mathfrak{m}_{\tau}^{3}$; this can be done with a standard basis of the ideal $\left(g_{2}\right)$. I will denote the set of exponents of these monomials by $B_{2}$ as well. Over $S_{2}$ one can solve (1.5): there are $\varphi_{\alpha} \in K^{1}$, with $\alpha \in B_{2}$, such that

$$
\sum_{\alpha \in B 2} t^{\alpha} d \varphi_{\alpha}+\frac{1}{2} \sum_{|i|=|j|=1} t^{i} t^{j}\left[\varphi_{i}, \varphi_{j}\right] \equiv 0 \bmod g_{2} .
$$

The $\varphi_{\alpha}$ are not unique, but determined up to elements $\psi_{\alpha} \in K^{1}$ with $d \psi_{\alpha}=0$. The indeterminacy can be reduced to a finite-dimensional one by choosing at the beginning a complement $C^{1}\left(K^{*}\right)$ to the coboundaries $B^{1}\left(K^{*}\right) \subset K^{1}$; the possible lifts 
then form a homogeneous space under $H^{1}\left(K^{\bullet}\right)$. If one uses an algorithm based on standard bases, the choices are unique (depending on the chosen monomial ordering).

For the next step one has to solve the equation

$$
\sum_{|\alpha|=3} t^{\alpha} d \varphi_{\alpha}+\sum_{\substack{|i|=1 \\ \alpha \in B_{2}}} t^{i} t^{\alpha}\left[\varphi_{i}, \varphi_{\alpha}\right] \equiv 0 \bmod g_{2} .
$$

Note that, although the ideal $\left(g_{2}\right)$ is defined in $\mathbb{C} \llbracket t] / \mathfrak{m}_{\tau}^{3}$, the ideal $\mathfrak{m}_{\tau}\left(g_{2}\right) \subset m_{\tau}^{3} / \mathfrak{m}_{\tau}^{4}$ is well-defined and does not depend on the extension of $\left(g_{2}\right)$ to $\mathbb{C} \llbracket t] / \mathfrak{m}_{\tau}^{4}$. The class of $\left[\varphi_{i}, \varphi_{\alpha}\right]$ in $H^{2}\left(K^{*}\right)$ is a Massey triple product. Write

$$
\operatorname{cl}\left(\left[\varphi_{i}, \varphi_{\alpha}\right]\right)=\sum_{k} c_{i \alpha}^{k} \Omega_{k}
$$

This gives

$$
g_{3}^{(k)}=\sum_{\substack{|i|=1 \\ \alpha \in B_{2}}} c_{i \alpha}^{k} t^{i} t^{\alpha}
$$

which defines the extension of $\left(g_{2}\right)$. Set $G_{3}^{(k)}=$ $g_{2}^{(k)}+g_{3}^{(k)}$, and define $S_{3}$ as the ring $\left.\mathbb{C}[t]\right] /\left(G_{3}\right)+$ $\mathfrak{m}_{\tau}^{4}$. Choose a basis $B_{3}$ of monomials for $\mathfrak{m}_{\tau}^{3} \cap$ $\left(\mathbb{C} \llbracket t \rrbracket /\left(G_{3}\right)+\mathfrak{m}_{\tau}^{4}\right)$. Over $S_{3}$ one can solve equation (1.4) using $\varphi_{\alpha}$ with $\alpha \in B_{3}$.

The notation becomes cumbersome, so I stop here, but the general procedure should be clear by now (see also [Laudal 1982]).

The number of nontrivial equations $G_{n}^{(k)}$ becomes stationary. As I am working with power series, the computation will in general be infinite, and therefore not suited for practical purposes. For deformations of singularities I will ensure finiteness by considering only deformations in negative degrees of weighted homogeneous germs. The convention here is that a deformation corresponds to a $\partial / \partial t_{i}$, so the parameters $t_{i}$ have positive weight, and therefore there is a bound on the possible exponents $\alpha$; indeed, the whole set-up reduces to a finite-dimensional one, and the process ends after a finite number of steps.

\section{DEFORMATIONS OF SINGULARITIES}

The definition of a deformation involves the notion of flatness, which accounts for the difficulties in explaining and understanding it. Mumford [1989] writes: 'The concept of flatness is a riddle that comes out of algebra, but which is technically the answer to many prayers.' Intuitively, in a flat family the fibres depend continuously on the points of the parametrising base space.

In this section all spaces considered are germs at the origin. Let $\pi: X \rightarrow S$ be a map of analytic germs, and suppose an embedding of the fibre $X_{0}=$ $\pi^{-1}(0) \subset \mathbb{C}^{N}$ is given. Then $\pi$ can be realised as the composition of an embedding $X \subset S \times \mathbb{C}^{N}$ and the projection of $S \times \mathbb{C}^{N}$ onto the first factor (for a proof see [Fischer 1976, 0.35]). In terms of equations one has generators $\left(F_{1}, \ldots, F_{k}\right)$ of the ideal $I$ of $X$ in $\mathcal{O}_{S}\{x\}$, while the $f_{i}(x):=F_{i}(0, x)$ generate the ideal $I_{0}$ of $X_{0}$ in $\mathcal{O}_{N}=\mathbb{C}\{x\}$.

Definition. The map $\pi: X \rightarrow S$ is flat in 0 , if every relation $\sum f_{i} r_{i}=0$ between the $f_{i}$ lifts to a relation $\sum F_{i} R_{i}=0 \in \mathcal{O}_{N}$ between the $F_{i}$.

It suffices to lift the generators of the module of relations. Consider a presentation of $\mathcal{O}_{X_{0}}$ :

$$
0 \longleftarrow \mathcal{O}_{X_{0}} \longleftarrow \mathbb{C}\{x\} \stackrel{f}{\longleftarrow}(\mathbb{C}\{x\})^{k} \stackrel{r}{\longleftarrow}(\mathbb{C}\{x\})^{l} .
$$

The entries of the row vector $f=\left(f_{1}, \ldots, f_{k}\right)$ generate the ideal $I_{0}$, and the columns of the matrix $r$ generate the module of relations. The flatness condition is that this presentation can be lifted to

$$
0 \longleftarrow \mathcal{O}_{X} \longleftarrow \mathcal{O}_{S}\{x\} \stackrel{F}{\longleftarrow}\left(\mathcal{O}_{S}\{x\}\right)^{k} \stackrel{R}{\longleftarrow}\left(\mathcal{O}_{S}\{x\}\right)^{l} .
$$

Example (complete intersections). If the map-germ $f$ : $\mathbb{C}^{n} \rightarrow \mathbb{C}^{k}$ defines a complete intersection $X_{0}$, the Koszul complex on the components $f_{i}$ of $f$ resolves the ring $\mathcal{O}_{X_{0}}$. In particular, the relations between the $f_{i}$ are generated by the obvious ones, $f_{i} f_{j}-$ $f_{j} f_{i}=0$, or, in vector notation,

$\left(f_{1}, \ldots, f_{i}, \ldots, f_{j}, \ldots, f_{k}\right) \cdot\left(0, \ldots, f_{j}, \ldots,-f_{i}, \ldots, 0\right)^{t}$ $=0$. 
For any deformation $F(t, x)$ of $f(x)$ one can lift the relation $f_{i} f_{j}-f_{j} f_{i}=0$ to the relation $F_{i} F_{j}-F_{j} F_{i}=$ 0 , and therefore the flatness condition is always satisfied.

Definition. A deformation of a germ $X_{0}$ is a flat map-germ $\pi: X \rightarrow S$ such that $X_{0}$ is isomorphic to the fibre $\pi^{-1}(0)$ under a given isomorphism $i$ : $X_{0} \rightarrow \pi^{-1}(0)$.

A morphism between deformations $\pi: X \rightarrow S$ and $\pi^{\prime}: X^{\prime} \rightarrow S^{\prime}$ of $X_{0}$ is a commutative diagram

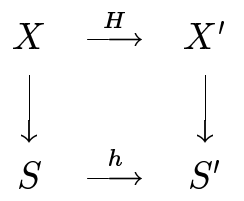

compatible with the embeddings $i: X_{0} \rightarrow X$ and $i^{\prime}: X_{0} \rightarrow X^{\prime}$, so $H \circ i=i^{\prime}$. I write in short $X \rightarrow X^{\prime}$, or, to emphasise the base spaces, $(X \rightarrow$ $S) \longrightarrow\left(X^{\prime} \rightarrow S^{\prime}\right)$.

Let $h: T \rightarrow S$ be a holomorphic map. The induced deformation is the flat map $h^{*}(\pi): X \times_{S}$ $T \rightarrow T$.

Definition. A deformation $\pi: X \rightarrow S$ of $X_{0}$ is called versal if it satisfies the following lifting property:

Let $(Y \rightarrow T) \longrightarrow\left(Y^{\prime} \rightarrow T^{\prime}\right)$ be a morphism of deformations, over an embedding $T \rightarrow T^{\prime}$. Then every morphism $(Y \rightarrow T) \longrightarrow(X \rightarrow S)$ can be lifted to a morphism $\left(Y^{\prime} \rightarrow T^{\prime}\right) \longrightarrow(X \rightarrow S)$ such that the composition $T \rightarrow T^{\prime} \rightarrow S$ equals $T \rightarrow S$.

A deformation is formally versal if the lifting property is satisfied for zero-dimensional spaces $T^{\prime}$. It is (formally) semi-universal if it is (formally) versal, and if the induced morphism $T_{0}\left(T^{\prime}\right) \rightarrow T_{0}(S)$ on the tangent spaces is uniquely determined (by $Y \rightarrow Y^{\prime}$ and $Y \rightarrow S$ ). For semi-universal the term miniversal is also used.

In particular, by taking $Y$ equal to $X_{0}$, one sees that every deformation $\rho: Y^{\prime} \rightarrow T^{\prime}$ of $X_{0}$ is isomorphic to a deformation $h^{*}(\pi)$, for some map $h: T^{\prime} \rightarrow S$. This property is often given as definition of versality. It is however not strong enough to prove that a versal deformation is the product of a miniversal one with a trivial factor [Flenner 1981, Lemma (5.3)]. This result allows one to speak (sloppily) about the versal deformation, i.e., to use the terminology of [Grauert 1972], where versal stands for miniversal, while versal is called complete. Sometimes it is easier to compute a versal but not miniversal deformation, if this leads to more symmetric formulas.

Grauert [1972] has shown the existence of versal deformations for isolated singularities. A formally versal formal object exists by the results of [Schlessinger 1968]. Every (holomorphic) deformation that is formally versal is already versal; this is a special case of a result of Flenner in a general deformation-theoretic context [Flenner 1981, Satz (5.2)]. In particular, a formal computation that happens to yield a convergent, or even polynomial, result already gives the versal family.

The method to compute versal deformations is the power series Ansatz of the previous Section. The relevant complex is the cotangent complex [Palamodov 1976], and the quadratic equation to be solved comes down to $F R=0$. Indeed, one can view the maps $f$ and $r$ as parts of a differential $d$ in a complex; if one resolves $\mathcal{O}_{X_{0}}$ with a differential graded algebra, the $T^{*}$ can be defined as the cohomology groups of its derivation algebra, in which the differential is given by $\delta u=[d, u]$. The deformation equation is obtained from the condition that the perturbed differential $d+\varphi$ gives again a complex: the equation $[d+\varphi, d+\varphi]=0$ yields

$$
\delta \varphi+\frac{1}{2}[\varphi, \varphi]=0
$$

For concrete computations it suffices to use direct, ad hoc definitions of $T^{1}$ and $T^{2}$ [Schlessinger 1973].

Since I am interested from now on in a fixed singularity, I will slightly change the notation and write $X$ for the germ that is deformed, and $I$ for its ideal in the power series ring $\mathcal{O}_{N}$.

Definition. A first-order infinitesimal deformation of $X$ is a deformation over the double point $\mathbb{D}$, the zero-dimensional space having as local ring the ring 
of dual numbers $\mathbb{C}[\varepsilon]:=\mathbb{C}[t] /\left(t^{2}\right)$. By definition $\varepsilon^{2}=0$.

Proposition 2.1. The $\mathcal{O}_{X}$-module of first-order deformations is isomorphic to the normal module $N_{X}=$ $\operatorname{Hom}_{X}\left(I / I^{2}, \mathcal{O}_{X}\right)$.

Proof. Let $F=f+\varepsilon f^{\prime}$ be an infinitesimal deformation, and let $R=r+\varepsilon r^{\prime}$ be a lift of the relations. Since $\varepsilon^{2}=0$, the flatness condition $F R=0$ gives

$$
\left(f+\varepsilon f^{\prime}\right)\left(r+\varepsilon r^{\prime}\right)=f r+\varepsilon\left(f r^{\prime}+f^{\prime} r\right)=0 .
$$

Because $f r=0$, one obtains the equation $f r^{\prime}+$ $f^{\prime} r=0$ in $\left(\mathcal{O}_{N}\right)^{l}$. The $\mathcal{O}_{N}$-homomorphism $\mathcal{O}_{N}{ }^{k} \rightarrow$ $\mathcal{O}_{N}$ that sends a generator $e_{i}$ to $f_{i}^{\prime}$ maps the image of $r$ into $I$, because $f^{\prime} r=-f r^{\prime}$. Hence $f^{\prime}$ induces a homomorphism

$$
\rho\left(f^{\prime}\right): \mathcal{O}_{N}^{k} / \operatorname{Im} r \cong I \rightarrow \mathcal{O}_{X}
$$

which sends $f_{i}$ to $\left(f_{i}^{\prime} \bmod I\right)$.

Conversely, given a homomorphism

$$
\varphi \in \operatorname{Hom}_{X}\left(I / I^{2}, \mathcal{O}_{X}\right) \cong \operatorname{Hom}_{\mathcal{O}_{N}}\left(I, \mathcal{O}_{X}\right),
$$

one lifts the vector

$$
\varphi(f)=\left(\varphi\left(f_{1}\right), \ldots, \varphi\left(f_{k}\right)\right) \in \mathcal{O}_{X}^{k}
$$

to a vector $f^{\prime} \in \mathcal{O}_{N}{ }^{k}$, inducing a homomorphism $\tilde{\varphi}: \mathcal{O}_{N}{ }^{k} \rightarrow \mathcal{O}_{N}$. For every relation $r_{j}$ the function $f^{\prime} r_{j}=\tilde{\varphi}\left(r_{j}\right)$ is a lift of $\varphi\left(\sum f_{i} r_{i j}\right)=0 \in \mathcal{O}_{X}$. Therefore one can find a matrix $r^{\prime}$ with $f^{\prime} r+f r^{\prime}=$ 0 . Any two liftings of $\varphi(f)$ differ by a $g \in I^{k}$, so they determine the same deformation.

An infinitesimal deformation $f+\varepsilon f^{\prime}$ is trivial if there is an automorphism $\varphi(x, \varepsilon)=(x+\varepsilon \delta(x), \varepsilon)$ of $\mathbb{C}^{N} \times \mathbb{D}$ such that $f+\varepsilon f^{\prime}$ and $f \circ \varphi$ determine the same ideal. Let $\Theta_{N}$ be module of germs of vector fields at the origin. The computation

$$
\begin{aligned}
\left.\frac{d}{d \varepsilon} f \circ \varphi(x, \varepsilon)\right|_{\varepsilon=0} & =\left.\frac{d}{d \varepsilon} f(x+\varepsilon \delta(x))\right|_{\varepsilon=0} \\
& =\sum_{j} \frac{\partial f}{\partial x_{j}} \delta_{j}(x)
\end{aligned}
$$

shows that the trivial deformations are the image of the natural map

$$
\left.\Theta_{N}\right|_{X}=\Theta_{N} \otimes \mathcal{O}_{X} \rightarrow \operatorname{Hom}_{\mathcal{O}_{N}}\left(I, \mathcal{O}_{X}\right)=N_{X},
$$

which sends a vector field $\delta$ to the homomorphism $g \mapsto \delta(g)$. The kernel of this map is the $\mathcal{O}_{X^{-}}$ module $\Theta_{X}=\left\{\left.\delta\right|_{X} \mid \delta(I) \subset I\right\}$. One has $\Theta_{X}=$ $\operatorname{Hom}_{X}\left(\Omega_{X}^{1}, \mathcal{O}_{X}\right)$.

Definition. The module $T_{X}^{1}$ of isomorphism classes of first-order infinitesimal deformations is

$$
T_{X}^{1}=\operatorname{coker}\left\{\left.\Theta_{N}\right|_{X} \rightarrow N_{X}\right\} .
$$

Remark. Suppose the singularity $X$ admits a good $\mathbb{C}^{*}$-action, so $\mathcal{O}_{X}$ is a (positively) graded module. Then all modules considered above inherit a grading.

Theorem 2.2 [Pinkham 1974, 2.3]. A singularity $X$ with good $\mathbb{C}^{*}$-action has a $\mathbb{C}^{*}$-equivariant versal deformation $\pi: X \rightarrow S$. The restriction $\pi_{-}: X_{-} \rightarrow$ $S_{-}$to the subspace of negative weight is versal for deformations of $X$ with negative weight.

Remark. For a given singularity, $T^{1}$ can be computed with a computer algebra package that computes standard bases and syzygies. The first step is to determine the $\mathcal{O}_{X}$-module $N_{X}$. Every generator $f+\varepsilon f^{\prime}$ satisfies $f^{\prime} r \equiv 0 \bmod I$. Taking the transpose gives $r^{t} f^{\prime t} \equiv 0 \bmod I$; in other words, $f^{\prime t}$ is a syzygy between the columns of the matrix $r^{t}$ over the ring $\mathcal{O}_{X}$ : the module $N_{X}$ is the syzygy module of the matrix $r^{t}$. The image of $\left.\Theta_{N}\right|_{X}$ is the submodule $J$ of $N_{X}$ generated by the partial derivatives of the row vector $f$. The resolution

$$
\mathcal{O}_{X}^{l} \stackrel{r^{t}}{\longleftarrow} \mathcal{O}_{X}{ }^{k} \stackrel{s_{1}}{\longleftarrow} \mathcal{O}_{X}^{s} \stackrel{s_{2}}{\longleftarrow} \mathcal{O}_{X}^{t}
$$

gives a presentation of $N_{X}$ :

$$
0 \longleftarrow N_{X} \longleftarrow \mathcal{O}_{X}^{k} \stackrel{s_{1}}{\longleftarrow} \mathcal{O}_{X}^{s} \stackrel{s_{2}}{\longleftarrow} \mathcal{O}_{X}^{t} .
$$

Lift the module $J$ to a submodule $\tilde{J}+\operatorname{Im} s_{2}$ of the free module $\mathcal{O}_{X}^{s}$ by lifting the generators: find a matrix $\tilde{\jmath}$ with $\partial f / \partial x=s_{1} \tilde{\jmath}$, and define $\tilde{J}$ as module generated by the columns of $\tilde{\jmath}$. Then $T_{X}^{1} \cong$ 
$\mathcal{O}_{X}^{s} /\left(\tilde{J}+\operatorname{Im} s_{2}\right)$. Choose representatives of a $\mathbb{C}$ basis of the quotient; to get a basis of $T^{1}$, which has a direct interpretation in terms of perturbations of the equations, take the image of the representatives under the map $s_{1}$.

I have written a script in Macaulay [Bayer and Stillman] for these computations (see section on Electronic Availability at the end of this article). Since Macaulay only computes in graded rings, the script can compute $T^{1}$ for singularities with good $\mathbb{C}^{*}$-action. For general singularities the program Singular [Greuel et al. 1994] will be useful.

Definition. As before, let $\mathcal{O}_{N} \stackrel{f}{\longleftarrow} \mathcal{O}_{N}{ }^{k} \stackrel{r}{\longleftarrow} \mathcal{O}_{N}{ }^{l}$ be a presentation of the ring $\mathcal{O}_{X}$. Let $\mathcal{R}=\mathcal{O}_{N}{ }^{l} / \operatorname{ker} r$ be the module of relations, and let $\mathcal{R}_{0}$ be the submodule of Koszul relations. Then $\mathcal{R} / \mathcal{R}_{0}$ is an $\mathcal{O}_{X^{-}}$ module. With these notations,

$T_{X}^{2}=\operatorname{coker}\left\{r^{t}: \operatorname{Hom}_{X}\left(\mathcal{O}_{X}^{k}, \mathcal{O}_{X}\right) \rightarrow \operatorname{Hom}_{X}\left(\mathcal{R} / \mathcal{R}_{0}, \mathcal{O}_{X}\right)\right\}$.

Example. For complete intersections one has $\mathcal{R}=$ $\mathcal{R}_{0}$, so $T^{2}=0$.

Proposition 2.3. Let $0 \rightarrow J \rightarrow A^{\prime} \rightarrow A \rightarrow 0$ be a small extension of Artinian rings (which means that $\left.J^{2}=0\right)$. The obstruction to extending a deformation of $X$ over $A$ to a deformation over $A^{\prime}$ lies in $T_{X}^{2} \otimes J$.

Proof. For notational convenience, I will consider one-parameter deformations: let $A=\mathbb{C}[t] /\left(t^{n}\right)$, and consider $F_{n-1}$ and $R_{n-1}$ with $F_{n-1} R_{n-1} \equiv 0$ $\bmod t^{n}$. I want a deformation over

$$
A^{\prime}=\mathbb{C}[t] /\left(t^{n+1}\right) .
$$

Write $F_{n}=F_{n-1}+t^{n} f^{(n)}$ and $R_{n}=R_{n-1}+t^{n} r^{(n)}$. The equation $F_{n} R_{n} \equiv 0 \bmod t^{n+1}$ gives

$$
F_{n-1} R_{n-1}+t^{n}\left(f^{(n)} r+f r^{(n)}\right) \equiv 0 \bmod t^{n+1} .
$$

Because $F_{n-1} R_{n-1} \equiv 0 \bmod t^{n}$, one has the equation

$$
t^{-n} F_{n-1} R_{n-1}+f^{(n)} r \equiv 0 \bmod t
$$

in $\mathcal{O}_{X}^{l}$. A simple computation shows that the vector $t^{-n} F_{n-1} R_{n-1} \bmod t \in \mathcal{O}_{X}^{l}$ (or, equivalently,
$\left.F_{n-1} R_{n-1} \bmod t^{n+1} \in\left(\mathcal{O}_{X} \cdot t^{n}\right)^{l}\right)$ depends only on $r$ (and $F_{n-1}$ ), but not on the lift $R_{n-1}$ of $r$. This vector represents an element of $\operatorname{Hom}_{\mathcal{O}_{N}}\left(\mathcal{O}_{N}{ }^{l}, \mathcal{O}_{X}\right)$, which vanishes on $\operatorname{ker} r$. The value of the homomorphism on any relation can be computed by taking a suitable lift $R^{\prime}$ of the particular relation. The canonical choice for a Koszul relation gives $F_{n-1} R^{\prime}=0 \in \mathcal{O}_{N}$. The equation $t^{-n} F_{n-1} R_{n-1}+$ $f^{(n)} r \equiv 0$ can be solved if and only if the homomorphism $t^{-n} F_{n-1} R_{n-1} \in \operatorname{Hom}\left(\mathcal{R} / \mathcal{R}_{0}, \mathcal{O}_{X}\right)$ can be lifted to the element of $\operatorname{Hom}\left(\mathcal{O}_{U}{ }^{k}, \mathcal{O}_{X}\right)$ given by the vector $f^{(n)}$.

To compute $T^{2}$, one first calculates the resolution of $\mathcal{O}_{X}$ one step further:

$$
0 \longleftarrow \mathcal{O}_{X} \longleftarrow \mathcal{O}_{N} \stackrel{f}{\longleftarrow}\left(\mathcal{O}_{N}\right)^{k} \stackrel{r}{\longleftarrow}\left(\mathcal{O}_{N}\right)^{l} \stackrel{s}{\longleftarrow}\left(\mathcal{O}_{N}\right)^{m} .
$$

Represent the Koszul relations by a $l \times\left(\begin{array}{l}k \\ 2\end{array}\right)$ matrix $k$; the concatenation $k s$ defines a presentation of $\mathcal{R} / \mathcal{R}_{0}$, and $\operatorname{Hom}_{X}\left(\mathcal{R} / \mathcal{R}_{0}, \mathcal{O}_{X}\right)$ is the syzygy module of $(k s)^{t}$ over $\mathcal{O}_{X}$. Dividing out the submodule generated by the columns of the matrix $r^{t}$ gives $T^{2}$. Also for these computations I have written a Macaulay script.

Example. Let $X$ be the the singularity consisting of two intersecting lines, with an imbedded component at the origin. In the same notation as above, the resolution of the local ring has the form

$$
0 \longleftarrow \mathcal{O}_{X} \longleftarrow \mathcal{O}_{3} \stackrel{f}{\longleftarrow}\left(\mathcal{O}_{3}\right)^{4} \stackrel{r}{\longleftarrow}\left(\mathcal{O}_{3}\right)^{4} \stackrel{s}{\longleftarrow} \mathcal{O}_{3} \longleftarrow 0,
$$

with matrices $f=\left(x y, x z, y^{2}, y z\right)$,

$$
r=\left(\begin{array}{rrrr}
0 & 0 & -y & -z \\
-y & 0 & 0 & y \\
0 & -z & x & 0 \\
x & y & 0 & 0
\end{array}\right)
$$

and $s=(y,-x,-z, y)^{t}$. The presentation of the normal module $N_{X}$ is given by the matrices

$$
s_{1}=\left(\begin{array}{cccccccc}
0 & 0 & 0 & 0 & 0 & y & x & y \\
0 & 0 & 0 & x & y & z & 0 & 0 \\
0 & 0 & y & 0 & 0 & 0 & 0 & 0 \\
y & z & 0 & 0 & 0 & 0 & 0 & 0
\end{array}\right)
$$


and a matrix $s_{2}$ consisting of the last 20 columns of the following concatenation:

$$
\begin{aligned}
& \left(\tilde{\jmath}, s_{2}\right)= \\
& \left(\begin{array}{ccccccccccccccccccccccc}
0 & 0 & 1 & 0 & 0 & 0 & 0 & 0 & 0 & 0 & 0 & 0 & 0 & 0 & 0 & 0 & 0 & 0 & 0 & -z & x & y & z \\
0 & 1 & 0 & 0 & 0 & 0 & 0 & 0 & 0 & 0 & 0 & 0 & 0 & 0 & 0 & 0 & 0 & 0 & x & y & 0 & 0 & 0 \\
0 & 2 & 0 & 0 & 0 & 0 & 0 & 0 & 0 & 0 & 0 & 0 & 0 & 0 & 0 & x & y & z & 0 & 0 & 0 & 0 & 0 \\
0 & 0 & 1 & 0 & 0 & 0 & 0 & 0 & -z & 0 & -y & 0 & 0 & y & z & 0 & 0 & 0 & 0 & 0 & 0 & 0 & 0 \\
0 & 0 & 0 & 0 & 0 & 0 & 0 & 0 & 0 & -z & x & y & z & 0 & 0 & 0 & 0 & 0 & 0 & 0 & 0 & 0 & 0 \\
1 & 0 & 0 & 0 & 0 & 0 & 0 & 0 & x & y & 0 & 0 & 0 & 0 & 0 & 0 & 0 & 0 & 0 & 0 & 0 & 0 & 0 \\
0 & 1 & 0 & -y & 0 & 0 & y & z & 0 & 0 & 0 & 0 & 0 & 0 & 0 & 0 & 0 & 0 & 0 & 0 & 0 & 0 & 0 \\
0 & 0 & 0 & x & y & z & 0 & 0 & 0 & 0 & 0 & 0 & 0 & 0 & 0 & 0 & 0 & 0 & 0 & 0 & 0 & 0 & 0
\end{array}\right) .
\end{aligned}
$$

It follows that

$$
\operatorname{dim} T^{1}=\operatorname{dim}\left(\left(\mathcal{O}_{X}\right)^{8} /\left(\tilde{J}+\operatorname{Im} s_{2}\right)\right)=5,
$$

and one can write as perturbed equations

$\left(x+s_{1}\right) y, \quad x z+y s_{2}, \quad y\left(y+s_{3}\right), \quad(y+t)\left(z+s_{4}\right)$.

A lift of the matrix $r$ is given by

$$
R_{1}=\left(\begin{array}{cccc}
-s_{4} & 0 & -y-s_{3} & -z \\
-y-t & 0 & 0 & y \\
s_{2} & -z-s_{4} & x+s_{1} & -s_{2} \\
x & y-t+s_{3} & 0 & s_{1}
\end{array}\right) .
$$

Modulo third-order terms one obtains the obstruction vector

$$
\begin{array}{r}
o=\left(x t s_{4}-y t s_{2}+y s_{2} s_{3}-y s_{1} s_{4},-z t\left(t-s_{3}\right), 0,\right. \\
\left.-y s_{2} s_{3}+y s_{1} s_{4}+z t s_{1}\right) .
\end{array}
$$

To find the class in $T^{2}$, one first computes the matrix $k s$, which describes $\mathcal{R} / \mathcal{R}_{0}$ :

$$
k s=\left(\begin{array}{rrrrrrr}
0 & 0 & 0 & 0 & -z & 0 & y \\
0 & 0 & -x & 0 & 0 & -y & -x \\
0 & -y & -z & -z & 0 & 0 & -z \\
-x & 0 & 0 & y & 0 & 0 & y
\end{array}\right) .
$$

Over $\mathcal{O}_{X}$ the syzygy matrix of the matrix $(k s)^{t}$ is

$$
\left(\begin{array}{cccccccc}
-y & 0 & 0 & x & 0 & x & y & 0 \\
0 & -z & 0 & y & z & 0 & 0 & 0 \\
0 & x & y & 0 & 0 & 0 & 0 & y \\
y & 0 & z & 0 & 0 & 0 & 0 & 0
\end{array}\right)
$$

A simple computation shows that $T^{2}$ is concentrated in degree -2 ; the first four columns of the above matrix are the columns of $r^{t}$ (in a different order), so the last four columns give a basis of $T_{X}^{2}$. In terms of these generators for $\operatorname{Hom}_{X}\left(\mathcal{R} / \mathcal{R}_{0}, \mathcal{O}_{X}\right)$ one finds the following expression for the obstruction vector:

$$
\begin{array}{r}
o=\left(-s_{2} s_{3}+s_{1} s_{4}, 0, t s_{1}, 0,-t\left(t-s_{3}\right),\right. \\
\left.t s_{4},-t s_{2},-t s_{1}\right) .
\end{array}
$$

The last four entries are the equations of the base space. The first four entries give the new quadratic terms of the equations of the total space. The final result is:

$$
\begin{aligned}
F=\left(\left(x+s_{1}\right) y, x z+\left(y+s_{3}\right) s_{2}-s_{1} s_{4},\right. \\
\left.y\left(y+s_{3}\right),(y+t)\left(z+s_{4}\right)\right) .
\end{aligned}
$$

One checks that $F R_{1} \equiv 0$ modulo the equations for the base space: $t s_{1}=t s_{2}=t\left(s_{3}-t\right)=t s_{4}=0$.

The deformation space has two components, a one-dimensional component $s_{1}=s_{2}=s_{3}-t=$ $s_{4}=0$ with

$$
(x y, x z, y(y+t),(y+t) z)
$$

as total space (two planes intersecting in one point) and a four-dimensional component where the embedded point can move off the curve.

\section{SMOOTHING COMPONENTS OF CURVES}

If the fibre $X_{s}$ over some point $s$ in the base space of a versal deformation $X \rightarrow S$ is smooth, $s$ is a regular point of $S$ (by openness of versality) and all nearby fibres are also smooth. In the terminology of [Wahl 1981] the irreducible component of $S$ containing $s$ is a smoothing component.

For a reduced curve singularity the dimension $e$ of smoothing components is an invariant of the singularity, and does not depend on the component. Deligne's formula for it simplifies for quasihomogeneous curves to $e=\mu+t-1$ [Greuel 1982]; here $t=\operatorname{dim} \omega / \mathfrak{m} \omega$ is the Cohen-Macaulay type, and the Milnor number $\mu$ can be computed as $\mu=$ 
$2 \delta-r+1$, with $r$ the number of branches and $\delta$ the number of virtual double points. Greuel used this formula to show that certain curve singularities are not smoothable.

Consider the curve $L_{r}^{n}$ consisting of $r$ general lines through the origin in $\mathbb{C}^{n}$; it is the affine cone over $r$ general points in $\mathbb{P}^{n-1}$. If $n<r \leq\left(\begin{array}{c}n+1 \\ 2\end{array}\right)$, then $\delta=2 r-n-1, t=r-n$ and $e=4 r-3 n-$ 2. The number of moduli of $r$ general points is $(n-1)(r-n-1)$; if the general curve is smoothable, the dimension of the smoothing component is greater than the number of moduli, giving

$$
(n-5)(r-n-2) \leq 6 .
$$

In this case every deformation $X_{t}$ of $X_{0}=L_{r}^{n}$ comes from a projective deformation $\bar{X}_{t}$ of $\bar{X}_{0}$, the projective cone over the $r$ points. If $\bar{X}_{t}$ is smooth, $\bar{X}_{t, \infty}$ is a hyperplane section (at infinity) of a smooth curve (of genus $g=r-n$ ) and $\bar{X}_{0, \infty}$, our set of $r$ points, is at least a limit of hyperplane sections. Pinkham arrived at the same bound $(n-5)(r-n-2) \leq 6$ by a dimension count in this context [Pinkham 1974, §11].

The number of moduli of $r$ points in $\mathbb{P}^{n-1}$ is equal to that of $r$ points in $\mathbb{P}^{r-n-1}$. In fact, the concept of association [Coble 1915] provides a birational isomorphism of moduli spaces of ordered point sets [Dolgachev and Ortland 1988, Chapter III]. In [Stevens 1989] I proved that a hyperplane section $\left\{p_{1}, \ldots, p_{r}\right\}$ of a nonspecial curve $C$ of genus $g$ in $\mathbb{P}^{r-g}$, and its image $\left\{\varphi\left(p_{1}\right), \ldots, \varphi\left(p_{r}\right)\right\}$ under the canonical embedding $\varphi_{K}: C \rightarrow \mathbb{P}^{g-1}$, are associated point sets. Therefore a set of $r$ general points in $\mathbb{P}^{n-1}$ is a hyperplane section (and the cone $L_{r}^{n}$ over it is smoothable) if and only if $r$ general points in $\mathbb{P}^{r-n-1}$ always lie on a canonical curve. Define $N(g)$ as the greatest number $r$ such that there is a canonical curve passing through $r$ general points in $\mathbb{P}^{g-1}$. The set of all pairs

$$
\left(C,\left\{p_{1}, \ldots, p_{r}\right\}\right)
$$

with $C$ a canonical curve of genus $g$ and $\left\{p_{1}, \ldots, p_{r}\right\}$ a set of $r$ distinct points in it, is an open subset of a certain Hilbert scheme, and there is a natural projection map to the Hilbert scheme parametrising point sets of degree $r$ in $\mathbb{P}^{g-1}$. The number $N(g)$ is the largest value of $r$ for which this map is generically surjective. An upper bound is obtained by computing the dimensions of the spaces involved. Not surprisingly, the result is the same as before; formulated in terms of $g$ it is $N(g) \leq$ $g+5+[6 / g-2]$. Surjectivity can be studied on the level of tangent spaces at points corresponding to singular curves. In this way I showed that $N(g) \geq g+5$ [Stevens 1989, Corollary 6].

For low values of $g$ the exact value of $N(g)$ is easily found:

$N(3)=14$ : plane quartics are determined by 14 points.

$N(4)=9$ : a canonical curve is the complete intersection of a cubic and a unique quadric. This quadric is determined by 9 points, while 6 additional points on it specify the curve.

$N(5)=12$ : the general curve is a complete intersection of three quadrics.

$N(6)=11$ : the general curve lies on a del Pezzo surface $S$ of degree 5 , which is determined by 11 points.

$N(7)=13$ : see $[$ Stevens 1989, 2.2], where a special, reducible curve is constructed.

In these examples $N(g)=g+5$ for $g$ even and $N(g)=g+5+[6 / g-2]$ for $g$ odd. This suggests for the remaining case $N(8)=13$. Despite great efforts I did not succeed in proving this. In fact, it turns out that the upper bound is obtained: $N(8)=14$; this follows from the solution of the original problem, namely, that a general $L_{14}^{6}$ is smoothable. For $r=14$ and $n=6$, equality holds in the formula $(n-5)(r-n-2) \leq 6$.

\section{THE VERSAL DEFORMATION OF $L_{14}^{6}$}

In this Section I describe the computations that show that the general $L_{14}^{6}$ is smoothable. Actually, I computed the versal deformation in negative degree for two different curves of type $L_{14}^{6}$, one with generic (random) coordinates, and afterwards 
a special one, which is more manageable, but still general enough. It is not possible to give details but I discuss separately the different stages of the computation. (See also the section on Electronic Availability at the end.)

\section{Step 0: The Equations of the Singularity}

The computations work with equations, but often they are not the most natural description of a singularity. In this case the starting point are the coordinates of the 14 points in $\mathbb{P}^{5}$. Since there are 35 moduli, it seemed out of the question to use variable coefficients. Instead, I produced a general curve $X$ with Macaulay by using random coordinates: from a $6 \times 14$ matrix, which was the concatenation of a $6 \times 6$ identity matrix and a random $6 \times 8$ matrix, the script points by David Eisenbud supplied 7 quadratic equations.

\section{Step 1: $T^{1}$}

In the computation one can take advantage of the fact that the matrix of relations has quadratic entries: among the 35 are the 21 Koszul relations. Before transposing the matrix $r$, I first determined a minimal set of generators of the module, generated over the ring $\mathcal{O}_{X}$ by the columns of $r$; this left me with a $14 \times 7$ matrix $r^{t}$. The dimension of $T^{1}$ is 43 ; in accordance with the results of [Greuel 1982, 3.5] and [Pinkham 1974, 11.1], there are no deformations in positive degree, and the degree 0 part has dimension 35 , which is the number of moduli of 14 points in $\mathbb{P}^{5}$. The surprising result was that $\operatorname{dim} T^{1}(-1)=8$; the dimension of a smoothing component is 36 .

In general, the basis of $T^{1}$ that comes out of the computation is not the most suitable to continue with; for example, for rational surface singularities the base space has at least one smooth component of maximal dimension, and it is advisable to have this as an intersection of coordinate hyperplanes. Therefore it is essential to analyse the results. In this case, due to the generic nature of the equations, every basis seems equally (un)suitable.
Step 2: $\mathrm{T}^{2}$

For $L_{14}^{6}$ one finds $\operatorname{dim} T^{2}=\operatorname{dim} T_{-2}^{2}=21$. Therefore one only has to compute the first obstruction. A second consequence is that the $\mathcal{O}_{X}$-module $T_{X}^{2}$ is annihilated by the maximal ideal, so there is no difference between generators of the module and generators of the $\mathbb{C}$-vector space.

Although generators of $T^{2}$ are needed, and will be computed again, over the ring in which the actual computation takes place (a ring with 14 variables in two blocks: 6 coordinates $x_{1}, \ldots, x_{6}$ and 8 deformation variables $\left.s_{1}, \ldots, s_{8}\right)$, the calculation of $T^{2}$ before setting up the computation of the versal deformation is useful to estimate its size. Since determining $T^{2}$ costs a lot of time and memory space, it is a good idea to stop standard basis and syzygy computations after the degree, in which the set of generators is complete. These degrees can be found at this stage.

\section{Step 3: The Actual Computation}

I compute the deformations only in negative degree, to ensure a finite calculation. This is not a severe restriction, as the transverse structure of the base space along the 35-dimensional equisingular locus will be (topologically) locally trivial for general moduli of the curve $L_{14}^{6}$ - this could define the term 'general'.

As in the example in Section 2, I form the row vector $f(x)+f^{\prime}(x, s)$ over the $\operatorname{ring} \mathbb{C}[x, s]$ : if $t$ is a $7 \times 8$ matrix representing $T^{1}(-1)$, then $f_{i}^{\prime}(x, s)=$ $\sum t_{i j} s_{j}$. I lift only 14 relations, which generate the module $\mathcal{R} / \mathcal{R}_{0}$, so $r(x)$ is a $7 \times 14$ matrix. Let $r^{\prime}(x, s)$ be a matrix with $f r^{\prime}+f^{\prime} r=0$.

Let $\mathfrak{m}_{s}$ be the maximal ideal of $\mathbb{C}[s]$, and let $J=\mathfrak{m}_{s}^{2} / \mathfrak{m}_{s}^{3}$. The transpose of the vector $f^{\prime} r^{\prime}$ represents an element of $\operatorname{Hom}_{X}\left(\mathcal{R} / \mathcal{R}_{0}, \mathcal{O}_{X}\right) \otimes J$, and projects onto an element of $T^{2} \otimes J$. To get an explicit expression, use a presentation of the module $\operatorname{Hom}_{X}\left(\mathcal{R} / \mathcal{R}_{0}, \mathcal{O}_{X}\right)$, computed over the quotient $\operatorname{ring} \mathbb{C}[x, s] /(f)$. Modulo the equations for the base space obtained by expressing $f^{\prime} r^{\prime}$ in a basis of $T^{2}$, I find a vector $f^{\prime \prime}(s)$, and $F=f+f^{\prime}+f^{\prime \prime}$ gives the 
versal family. The total computation takes about an hour on a Mac SE 30, and needs 1.5 Megabytes of memory.

\section{Step 4: Analysis of the Results}

I do not reproduce here equations of the base space $S$ of the versal deformation in negative degrees; there are 21 quadratic equations (in 8 variables), which contain together 336 monomials - the maximal number for a standard basis. The coefficients give the impression of being 'random'. The ideal of $S$ has degree 16 and codimension 7 . With the jacobian criterion one finds that $S$ is smooth outside the origin; in fact it suffices to compute the first minor of the jacobian matrix to conclude that $S$ is a cone over 16 distinct points; these points are not in general position: they impose one condition less on quadrics. The points form a self-associated point set in $\mathbb{P}^{7}$; such sets depend on 28 moduli [Dolgachev and Ortland 1988, Theorem III.4].

All 16 components of the base space are smoothing components, as can be seen by computing the relative critical locus; it suffices to take $3 \times 3$ minors of the matrix $\partial F(x, s) / \partial x$, because all curve singularities in $\mathbb{C}^{3}$ are smoothable (computing with $5 \times 5$ minors takes too long). It could be that the 'random' singularity is not generic, but special. Nevertheless, one can draw conclusions about the general curve, because the base space $T$ of the versal deformation (in all degrees) has a $\mathbb{C}^{*}$ action with weights 0 and 1 , which defines a projection of $T$ onto the degree 0 part.

Proposition 4.1. A general curve singularity $L_{14}^{6}$ is smoothable, and has 16 smoothing components of dimension 36 , while $\operatorname{dim} T^{1}=43$.

The curve $L_{14}^{6}$ gives, to my knowledge, the first example of a curve singularity with several smoothing components. As the moduli of the curve enter in the equations of the base space, it is hopeless to find irreducible components and to actually write down a smoothing for a generic curve. But it is possible to compute with a special $L_{14}^{6}$ that is still 'general enough' and get explicit equations for one-parameter deformations. I follow the same programme as before.

\section{Step 0: The Equations of the Singularity}

I start by looking for a singularity defined by oligonomials [Khovanskii 1984]: polynomials in which only a few monomials occur. The hope is that this property persists through the entire computation, which is therefore shorter and faster, and that the final results are more manageable. The first idea, to take the 6 coordinate axes and the cone over 8 points on a rational normal curve, does not work, because the ideal is then generated by quadrics and cubics. Instead, consider 6 coordinate points, 6 points $s^{6}-t^{6}$ on the curve $\left(s^{6}, s^{5} t\right.$, $\left.\ldots, t^{6}\right)$, and two other points; after some experimentation I took $(1,1,0,1,0,1)$ and $(1,0,1,0,1,1)$. The first 12 points give a $L_{12}^{6}$ with particular nice equations:

$$
x_{i} x_{i+3}-x_{i+1} x_{i+2} \quad \text { and } \quad x_{i-1} x_{i+1}-x_{i-2} x_{i+2},
$$

where $i \in \mathbb{Z} / 6 \mathbb{Z}$. This curve has only equisingular deformations: a computation reveals that $T^{1}$ is concentrated in degree 0 . This shows again that $N(6)=11$.

\section{Steps 1-3}

Again $\operatorname{dim} T^{1}(-1)=8$. Some simplification of the matrix for $T^{1}$ is possible, but it is not clear what the simplest form is. For the rest the computation is similar to the general case, and somewhat faster (forty minutes on a Mac SE 30).

\section{Steps 4: Analysis of the Results}

The equations for the base space and the total space are still rather complicated. In order to simplify them, I first computed the primary decomposition of the ideal $J$ of the base space $S$. This can be done by computing ideal quotients $(J: l)$ for various linear functions $l$; since there are 21 equations, and $J$ has degree 3 and dimension 2 (easily computed from a standard basis), one quickly finds some components. A coordinate transformation placing the components as far as possible in 
the intersection of coordinate hyperplanes gives the following equations for $S$ :

$s_{2} s_{1}, \quad s_{3} s_{4}, \quad s_{2} s_{3}, \quad s_{2} s_{8}, \quad s_{3} s_{5}, \quad s_{1} s_{5}, \quad s_{4} s_{8}, \quad\left(3 s_{2}+s_{5}\right) s_{7}, \quad\left(3 s_{3}+s_{8}\right) s_{6}, \quad s_{2}\left(s_{5}+3 s_{6}\right), \quad s_{3}\left(s_{8}+3 s_{7}\right)$, $s_{2}\left(6 s_{4}-2 s_{6}+3 s_{7}\right), \quad s_{3}\left(6 s_{1}-2 s_{7}+3 s_{6}\right), \quad s_{4}\left(6 s_{4}+3 s_{7}-2 s_{6}-4 s_{1}\right), \quad s_{1}\left(6 s_{1}+3 s_{6}-2 s_{7}-4 s_{4}\right), \quad\left(2 s_{1}-2 s_{3}+s_{6}\right) s_{8}$, $\left(2 s_{4}-2 s_{2}+s_{7}\right) s_{5}, \quad\left(2 s_{2}-2 s_{4}-s_{7}+s_{8}\right) s_{6}, \quad\left(2 s_{3}-2 s_{1}-s_{6}+s_{5}\right) s_{7}, \quad s_{1} s_{4}-s_{6} s_{7}, \quad s_{5} s_{7}+s_{6} s_{8}+3 s_{6} s_{7}$.

After further coordinate transformations on the total space the equations contain only 124 monomials; this number can be brought down, at the cost of complicating the equations for $S$. The equations are not very enlightening, but I write them down to show how complicated things can get, even in favourable circumstances.

$$
\begin{aligned}
& x_{3} x_{4}-x_{2} x_{5}-s_{6} s_{7}, \quad x_{2} x_{3}-x_{4} x_{5}+x_{3} x_{6}-x_{5} x_{6}-x_{2} s_{1}-x_{2} s_{6}-x_{3} s_{7}+x_{4} s_{6}+x_{5} s_{7}+x_{6} s_{1}+2 s_{3} s_{7}, \\
& -x_{1} x_{2}+x_{1} x_{4}+x_{3} x_{6}-x_{5} x_{6}+x_{1} s_{4}-x_{2} s_{1}-x_{5} s_{4}+x_{6} s_{1}+2 s_{2} s_{6}+2 s_{3} s_{7}, \\
& x_{1} x_{2}-x_{2} x_{3}+x_{2} x_{5}-x_{4} x_{5}-x_{1} x_{6}+x_{5} x_{6}-2 x_{1} s_{4}-2 x_{1} s_{7}+2 x_{2} s_{1}+x_{2} s_{6} \\
& +x_{3} s_{7}+x_{4} s_{6}+2 x_{5} s_{4}+x_{5} s_{7}-2 x_{6} s_{1}-2 x_{6} s_{6}-6 s_{1} s_{4}-4 s_{4} s_{6}-4 s_{1} s_{7}, \\
& x_{1} x_{2}+x_{1} x_{3}-x_{3} x_{6}-x_{4} x_{6}-x_{1} s_{2}-x_{1} s_{1}-x_{1} s_{6}+x_{2} s_{1}-\frac{3}{2} x_{3} s_{3}-\frac{1}{2} x_{3} s_{7} \\
& -x_{3} s_{8}+\frac{3}{2} x_{4} s_{2}+x_{4} s_{5}+\frac{1}{2} x_{4} s_{6}-x_{6} s_{1}+x_{6} s_{3}+x_{6} s_{4}+x_{6} s_{7}-2 s_{3} s_{7}, \\
& x_{1} x_{2}+x_{3} x_{5}-x_{2} x_{6}-x_{3} x_{6}-\frac{4}{3} x_{1} s_{1}-\frac{5}{6} x_{1} s_{3}-\frac{2}{3} x_{1} s_{6}-\frac{1}{2} x_{1} s_{7}-x_{1} s_{8}+\frac{3}{2} x_{2} s_{2}-x_{2} s_{3}+x_{2} s_{5}+\frac{1}{2} x_{2} s_{6}-\frac{4}{3} x_{3} s_{1}+\frac{2}{3} x_{3} s_{3}-x_{3} s_{4} \\
& -\frac{5}{3} x_{3} s_{6}-x_{5} s_{2}-\frac{4}{3} x_{5} s_{1}+\frac{2}{3} x_{5} s_{3}+x_{5} s_{4}-\frac{2}{3} x_{5} s_{6}-x_{6} s_{3}+x_{6} s_{4}+x_{6} s_{7}+2 s_{2} s_{6}+\frac{4}{3} s_{1} s_{6}-\frac{13}{6} s_{3} s_{6}+\frac{2}{3} s_{6}^{2}+2 s_{1} s_{7}+5 s_{6} s_{7}, \\
& -x_{1} x_{4}+x_{2} x_{4}-x_{1} x_{5}+x_{5} x_{6}-x_{1} s_{2}+x_{1} s_{1}+x_{1} s_{6}+\frac{2}{3} x_{2} s_{2}+x_{2} s_{1}-x_{2} s_{3}-\frac{4}{3} x_{2} s_{4} \\
& -\frac{2}{3} x_{2} s_{7}+\frac{2}{3} x_{4} s_{2}-x_{4} s_{1}-\frac{4}{3} x_{4} s_{4}-\frac{5}{3} x_{4} s_{7}-x_{5} s_{2}+\frac{3}{2} x_{5} s_{3}+\frac{1}{2} x_{5} s_{7}+x_{5} s_{8}-\frac{5}{6} x_{6} s_{2} \\
& -\frac{4}{3} x_{6} s_{4}-x_{6} s_{5}-\frac{1}{2} x_{6} s_{6}-\frac{2}{3} x_{6} s_{7}+2 s_{4} s_{6}-\frac{13}{6} s_{2} s_{7}+2 s_{3} s_{7}+\frac{4}{3} s_{4} s_{7}+5 s_{6} s_{7}+\frac{2}{3} s_{7}^{2} \text {. }
\end{aligned}
$$

The $\mathbb{Z} / 2 \mathbb{Z}$-symmetry on the singularity induces the involution $\left(s_{1}, \ldots, s_{8}\right) \mapsto\left(s_{4}, s_{3}, s_{2}, s_{1}, s_{8}, s_{7}, s_{6}, s_{5}\right)$ on the base space $S$. There are nine components. Three of them have dimension two, $C_{7}:$ the $\left(s_{7}, s_{8}\right)$-plane, $C_{8}$ : the $\left(s_{5}, s_{8}\right)$-plane, and $C_{9}$ : the $\left(s_{5}, s_{6}\right)$-plane; $C_{8}$ intersects $C_{7}$ and $C_{9}$ in a line, while $C_{7} \cap C_{9}=\varnothing$. The other components have dimension one; four of them are reduced, while $C_{5}$ and $C_{6}$ have a multiplicity two structure. The general fibre over each of these components is reducible.

Each line $L$ in $S$ defines a one parameter deformation $X \rightarrow L$ of $X$; all fibres $X_{l}$ with $l \neq 0$ are isomorphic, and the projective closure of $X_{l}$ is isomorphic to the projective curve, defined in $\mathbb{P}^{7}=\mathbb{P}\left(\mathbb{C}^{6} \times L\right)$ by the equations of $X$.

The total space over the component $C_{8}$ is given by the equations

$$
\begin{aligned}
& x_{3} x_{4}-x_{2} x_{5}, \quad x_{2} x_{3}-x_{4} x_{5}+x_{3} x_{6}-x_{5} x_{6}, \quad-x_{1} x_{2}+x_{1} x_{4}+x_{3} x_{6}-x_{5} x_{6}, \quad-x_{1} x_{4}+x_{2} x_{4}-x_{1} x_{5}+x_{5} x_{6}+x_{5} s_{8}-x_{6} s_{5}, \\
& x_{1} x_{2}+x_{1} x_{3}-x_{3} x_{6}-x_{4} x_{6}-x_{3} s_{8}+x_{4} s_{5}, \quad x_{1} x_{2}+x_{3} x_{5}-x_{2} x_{6}-x_{3} x_{6}-x_{1} s_{8}+x_{2} s_{5}, \quad x_{1} x_{2}-x_{2} x_{3}+x_{2} x_{5}-x_{4} x_{5}-x_{1} x_{6}+x_{5} x_{6} .
\end{aligned}
$$

It has seven components of degree two, given by

$$
\begin{array}{lll}
\left(x_{4}-x_{6}, x_{3}-x_{5}, x_{2}-x_{6}, x_{5}+s_{8},\left(x_{1}+x_{5}\right) x_{5}+\left(x_{1}-x_{5}-x_{6}+s_{5}\right) x_{6}\right), & \left(x_{1}, x_{3}, x_{5}, x_{6}-s_{5}, x_{2} x_{4}-x_{6}^{2}\right), \\
\left(x_{1}-x_{3}, x_{2}-x_{4}, x_{1}-x_{5}, x_{2}+s_{5}, x_{2}\left(x_{2}+x_{6}\right)+x_{1}\left(x_{6}-x_{1}-x_{2}+s_{8}\right)\right), & \left(x_{2}, x_{4}, x_{6}, x_{1}-s_{8}, x_{3} x_{5}-x_{1}^{2}\right), \\
\left(x_{4}-\omega x_{6}, x_{2}-\omega^{2} x_{6}, x_{3}-\omega^{2} x_{1}, x_{5}-\omega x_{1}, x_{1}\left(x_{1}-s_{8}\right)-\omega^{2} x_{6}\left(x_{6}-s_{5}\right)\right), &
\end{array}
$$

where $\omega^{3}=1$ in the last expression (so it stands for three quadrics). Over the intersection with the component $C_{7}$ one has $s_{5}=0$, so the second and third quadric are reducible. The projective curve now consists of four lines:

$$
\begin{array}{ll}
i_{1}=\left(x_{1}, x_{2}, x_{3}, x_{4}, x_{5}\right), & i_{2}=\left(x_{1}-x_{3}, x_{2}, x_{4}, x_{1}-x_{5}, x_{6}-x_{1}+s_{8}\right), \\
i_{3}=\left(x_{1}, x_{2}, x_{3}, x_{5}, x_{6}\right), & i_{4}=\left(x_{1}, x_{3}, x_{4}, x_{5}, x_{6}\right)
\end{array}
$$


and five conics:

$$
\begin{aligned}
& i_{5}=\left(x_{4}-x_{6}, x_{3}-x_{5}, x_{2}-x_{6}, x_{5}+s_{8},\right. \\
&\left.\left(x_{1}-x_{6}\right)\left(x_{5}+x_{6}\right)+x_{5}^{2}\right), \\
& i_{6}=\left(x_{1}-s_{8}, x_{2}, x_{4}, x_{6}, x_{1}^{2}-x_{3} x_{5}\right), \\
& i_{7}, i_{8}, i_{9}=\left(x_{4}-\omega x_{6}, x_{2}-\omega^{2} x_{6}, x_{3}-\omega^{2} x_{1},\right. \\
&\left.x_{5}-\omega x_{1}, x_{1}\left(x_{1}-s_{8}\right)-\omega^{2} x_{6}^{2}\right) .
\end{aligned}
$$

Through the point $P=(0: 0: 0: 0: 0: 0: 1)$ pass the lines $i_{1}, i_{2}, i_{3}$, and all conics. In affine coordinates the lines are the coordinate axes in the three-dimensional $\left(x_{2}, x_{4}, x_{6}\right)$-space, while the tangent to a conic is given by $x_{2}=\omega^{2} x_{6}, x_{4}=\omega x_{6}$; this gives six lines not on a quadric cone, so $P$ is a singular point of type $L_{6}^{3}$. The intersection $(0:-1: 0:-1: 1: 0: 1)$ of $i_{4}, i_{6}$ and $i_{7}$ is of type $L_{3}^{3}$. Finally there are 6 ordinary double points: $i_{3}$ intersects $i_{4}, i_{6}$ intersects $i_{5}, i_{8}$ and $i_{9}$, while $i_{5}$ intersects $i_{7}$ in two points. The curve has indeed arithmetic genus 8 .

The embedding dimension of the singular points is at most 3. This implies that the base space of the versal deformation (in all degrees) is smooth in a general point of $C_{7} \cap C_{8}$. The same is true over $C_{8} \cap C_{9}$. Therefore these three components of $S$ lie in the same smoothing component. This shows that one has to be careful, when drawing conclusion from the negative degree part about the versal base. What happens here can be understood in the following way: the three components together are the cone over a curve of degree 3 of arithmetic genus 0 ; the ideal of such a curve is generated by 3 quadrics, yoked together by 2 linear relations. A general (non flat) perturbation of the quadrics gives a complete intersection, consisting of 8 points, and if the number of deformation parameters is large enough, the total space will be smooth. The base space is not normally flat along the equisingular locus.

The double component $C_{5}$ has ideal

$\left(s_{8}, s_{5}+3 s_{6}, 2 s_{4}-s_{1}+s_{7}, s_{3}, s_{1}+2 s_{6}, s_{2}-s_{6}, s_{6}^{2}\right)$,

so its reduction is the line $2 s_{4}+s_{7}=0$ in the $\left(s_{4}, s_{7}\right)$-plane. The total space over this line has 7 components; it is the cone over a curve with one singular point of type $L_{3}^{3}$, and 12 ordinary double points. Therefore this curve represents also a smooth point of the base of the versal deformation. A model for the smoothing component in question is a pinch point times a smooth factor. The same holds for the other double component of $S$.

Proposition 4.2. The special $L_{14}^{6}$ considered above has 7 smoothing components.

\section{REMARKS}

1. It seems to be well known that the complexity of the basic algorithms is doubly exponential. In practical applications one observes that the size and length of computations grows rapidly, say as the codimension of the singularity increases. The same tends to be true for the results of computations. Beyond the cases one can compute by hand, there is a small number of examples open to computer treatment, and after that the problems are too large. To get results, it is important to try to limit the size. This is also the reason that I do not have a Macaulay script for the computation of versal deformations, in contrast with $T^{1}$ and $T^{2}$; I need control over the progress of a computation, and, if necessary, need to be able to exploit special properties of a specific example.

A good approach is to compute only for singularities with special moduli; the case of $L_{14}^{6}$ shows that this strategy has to be used with caution. Candidates are singularities with extra symmetry. Although the symmetry does not enter directly into machine calculations, it is important for two reasons. Firstly, since an equivariant deformation exists [Rim 1980], there are constraints on the monomials, occurring in the equations. Secondly, the extra structure makes it easier to give an interpretation of the results.

Even without symmetry, one should look for singularities defined by oligonomials. There is no guarantee that the versal deformation is also defined by oligonomials, but in many cases this happens. The toy example of Section 2 is defined by 
monomials, but there is no coordinate transformation, which makes the total space over the largest component monomial.

2. It is not necessary to lift all relations. It is clear that one can ignore Koszul relations. Less obvious is the case of determinantal singularities. Let the equations of $X$ consist of the $\left(\begin{array}{l}n \\ 2\end{array}\right)$ minors of a $2 \times n$ matrix:

$$
\left(\begin{array}{lll}
f_{1} & \ldots & f_{n} \\
g_{1} & \ldots & g_{n}
\end{array}\right)
$$

Each $3 \times 3$ minor of the $3 \times n$ matrix

$$
\left(\begin{array}{lll}
f_{1} & \ldots & f_{n} \\
f_{1} & \ldots & f_{n} \\
g_{1} & \ldots & g_{n}
\end{array}\right)
$$

gives a relation, and since one can also repeat the second row, this construction gives $2\left(\begin{array}{l}n \\ 3\end{array}\right)$ relations, which together generate the module of relations if $X$ is Cohen-Macaulay of codimension $n-1$ (only in this case is $X$ called determinantal). To compute deformations it suffices to lift the $\left(\begin{array}{l}n \\ 3\end{array}\right)$ relations obtained by doubling only one row. For small $n$ it is not worthwhile to single out these relations, but for larger $n$ one obtains a significant simplification.

3. Macaulay computes only in finite fields. This poses the problem of lifting the results back to characteristic zero. In the example of the special $L_{14}^{6}$ the coefficients in the final equations (after my coordinate transformations) have denominator at most 6 , and numerator at most 13 ; the computation was done in characteristic 31991, which is large enough to safely assume the validity of the result. In intermediate steps large coefficients do occur.

In other situations one can repeat the computation in a different characteristic. Here again the course to follow depends on the results; equations with large (integer) coefficients are less useful, unless an interpretation of these coefficients exists.

4. A second restriction of Macaulay is that it can only compute standard bases of quasihomogeneous modules. Singular [Greuel et al. 1994] will work in power series rings, so it will be able to compute $T^{1}$ and $T^{2}$ for nongraded singularities. As to the computation of versal deformations in the general case, there is no guarantee that the process stops after a finite number of steps. In some cases a (weighted) tangent cone gives enough information about the structure of the base space, so a computation up to low powers of the deformation variables will suffice.

5. According to the algorithm, as described, one has to express the obstruction vector in a $\mathbb{C}$-basis of $T^{2}$. With Macaulay one finds an expression in terms of $\mathcal{O}_{X}$-generators of $T^{2}$. As a $\mathbb{C}$-basis consists of monomial multiples of $\mathcal{O}_{X^{-}}$generators, it suffices to take coefficients.

6. In the obstruction calculus one has to reduce expressions modulo the ideals $\left(G_{m}\right)+\mathfrak{m}_{\tau}^{m+1}$. As the number of generators of $\mathfrak{m}_{\tau}^{m+1}$ tends to be large, finding a standard basis will take a long time. A useful trick is to replace the deformation parameters $s_{i}$ by $\varepsilon \sigma_{i}$, with $\varepsilon$ a new parameter (of weight 1 ; take wt $\sigma_{i}=2$ wt $s_{i}-1$, and multiply the weights of the $x_{i}$ by 2). It now suffices to reduce modulo the ideal, generated by $G_{m}(\varepsilon \sigma)$ and $\varepsilon^{m+1}$. On the other computations this substitution has hardly any influence.

\section{ACKNOWLEDGEMENT}

I thank Jan Christophersen and Duco van Straten for introducing me to the computation of deformations with Macaulay; the scripts I wrote for the computation of $T^{1}$ and $T^{2}$ (available upon request) are based on a script by Jan Christophersen.

\section{REFERENCES}

[Bayer and Stillman] Dave Bayer and Mike Stillman, Macaulay: A system for computation in algebraic geometry and commutative algebra. Computer software, available via anonymous ftp from zariski. harvard.edu.

[Coble 1915] Arthur B. Coble, "Point sets and allied Cremona groups I", Trans. Amer. Math. Soc. 16 (1915), 155-198. 
[Dolgachev and Ortland 1988] Igor Dolgachev and David Ortland, Point sets in projective spaces and theta functions, Astérisque 165, Soc. Math. de France, Paris, 1988.

[Fischer 1976] Gerd Fischer, Complex Analytic Geometry, Lecture Notes in Math. 538, Springer, Berlin, 1976.

[Flenner 1981] Hubert Flenner, "Ein Kriterium für die Offenheit der Versalität", Math. Z. 178 (1981), 449473.

[Goldman and Millson 1990] William M. Goldman and John J. Millson, "The homotopy invariance of the Kuranishi space", Illinois. J. Math. 34 (1990), 337367.

[Grauert 1972] Hans Grauert, "Über die Deformationen isolierter Singularitäten analytischer Mengen", Invent. Math. 15 (1972), 171-198.

[Greuel 1982] Gert-Martin Greuel, "On deformations of curves and a formula of Deligne", pp. 141-168 in Algebraic Geometry, La Rábida, Spain, 1981 (edited by J. M. Aroca et al.), Lecture Notes in Math. 961, Springer, Berlin, 1982.

[Greuel et al. 1994] G.-M. Greuel, G. Pfister and H. Schönemann, Singular: A computer algebra system for singularity theory and algebraic geometry, with contributions from H. Grassmann, W. Neumann, W. Pohl and T. Siebert. Software, available via anonymous ftp from www.mathematik.uni-kl.de.

[Griffiths 1965] Phillip A. Griffiths, "The extension problem for compact submanifolds of complex manifolds I: The case of a trivial normal bundle", pp. 113142 in Proceedings (Conference on Complex Analysis, Minneapolis, 1964; edited by A. Aeppli et al.), Springer, Berlin, 1965.

[Khovanskii 1984] A. G. Khovanskii, "Fewnomials and Pfaff manifolds", pp. 549-564 in Proc. Int. Congress of Math., Warszawa, 1983, vol. I (edited by Z. Ciesielski and Cz. Olech), PWN, Warszawa, and North-Holland, Amsterdam, 1984.
[Kuranishi 1971] Masatake Kuranishi, "Deformations of compact complex manifolds", Sém. de math. sup. 39, Presses de l'univ. de Montréal, 1971.

[Laudal 1982] O. A. Laudal, "Matric Massey products and formal moduli, I", pp. 141-168 in Algebra, Algebraic Topology and their Interactions, Stockholm, 1983 (edited by J.-E. Roos), Lecture Notes in Math. 1183, Springer, Berlin, 1986.

[Mumford 1989] David Mumford, The Red Book of Varieties and Schemes, Lecture Notes in Math. 1358, Springer, Berlin, 1989.

[Nijenhuis 1969] Albert Nijenhuis, "On a class of common properties of some different types of algebras", Nieuw. Arch. Wisk. (3) 17 (1969), I: 17-46, II: 87108.

[Palamodov 1976] V. P. Palamodov, "Deformations of Complex Spaces", Russian Math. Surveys 31:3 (1976), 129-197.

[Pinkham 1974] Henry C. Pinkham, Deformations of algebraic varieties with $G_{m}$-action, Astérisque 20, Soc. Math. de France, Paris, 1974.

[Rim 1980] Dock S. Rim, "Equivariant $G$-structure on versal deformations", Trans. Amer. Math. Soc. 257 (1980), 217-226.

[Schlessinger 1968] Michael Schlessinger, "Functors of Artin rings", Trans. Amer. Math. Soc. 130 (1968), 208-222.

[Schlessinger 1973] Michael Schlessinger, "On rigid singularities", Rice Univ. Stud. 59 (1973), 147-162.

[Stevens 1989] Jan Stevens, "On the number of points determining a canonical curve", Indag. Math. 51 (1989), 485-494.

[Wahl 1981] Jonathan Wahl, "Smoothings of normal surface singularities", Topology 20 (1981), 219-240.

\section{ELECTRONIC AVAILABILITY}

The interested reader can obtain my Macaulay scripts referred to above, as well as the complete files containing the calculation in Section 4, by writing to me. 
144 Experimental Mathematics, Vol. 4 (1994), No. 2

Jan Stevens, Mathematisches Seminar der Universität Hamburg, Bundesstraße 55, D 20146 Hamburg, Germany (stevens@math.uni-hamburg.de)

Current address (from October 1995): Matematiska institutionen, Chalmers tekniska högskola, S-41296 Göteborg, Sweden

Received March 19, 1995; accepted in revised form June 19, 1995 